\title{
PHYSIOLOGICAL ANALYSIS OF BICOZAMYCIN HIGH-PRODUCING STREPTOMYCES GRISEOFLAVUS USED AT INDUSTRIAL LEVEL
}

\author{
Kozo Ochi, Yasuhisa Tsurumi, Nobuharu Shigematsu, Morita Iwam, \\ KaZUYOShI UMEHARA and MASAKUNI OKUHARA \\ Exploratory Research Laboratories, Fujisawa Pharmaceutical Co., Ltd., \\ 5-2-3 Tokodai, Tsukuba-city, Ibaraki 300-26, Japan \\ (Received for publication February 4, 1988)
}

\begin{abstract}
Streptomyces griseoflavus, a bicozamycin-producing wild type strain and its highproducing one derived from it by multiple $(>15)$ mutagenic treatments, were analyzed physiologically and biochemically. The high-producing strain was characterized by: (1) An increased pool size of amino acids including leucine and isoleucine, precursors for bicozamycin synthesis, (2) an earlier and greater accumulation of intracellular ppGpp, (3) a more accentuated decrease in GTP pool size, (4) a higher specific activity of ornithine transcarbamylase which produces citrulline, (5) an increased ability to form aerial mycelium, and (6) an increased resistance to its own antibiotic. We propose that (1), (2) and (4) may be responsible for the high yields of bicozamycin and, possibly, of some other antibiotics produced by Streptomyces sp.
\end{abstract}

One of the distinguishing properties of the genus Streptomyces is their abilities to differentiate (i.e. aerial mycelium formation and sporulation) and to produce numerous antibiotics. ${ }^{1,2}$ Differentiation and secondary metabolism usually occur in parallel in response to nutrient limitation. ${ }^{3,4}$ It seems reasonable, therefore, to assume that these two phenomena may partially share regulatory mechanisms, especially with regard to their initiation processes. The pleiotropic loss of multiple functions has been ascribed to a loss of autoregulatory effectors (e.g. A-factor, ${ }^{5 \sim 7)}$ factor $\mathrm{C}^{8)}$ ) and arginine auxotrophy. ${ }^{9 \sim 11}$ Although it is recognized that some mutations result in block of cytodifferentiation, ${ }^{12,132}$ the biochemical basis for this derangement remains unknown. By isolating and analyzing the relaxed ( $\mathrm{rel}$ ) mutants of several Streptomyces sp. OCHI has stressed the significance of the stringent response (ppGpp) for their morphological and physiological differentiation. ${ }^{14 \sim 17)}$ The importance of the metabolic control of Streptomyces differentiation has been well documented by COLEMAN and ENSIGN, ${ }^{18)}$ and VARGHA et al. ${ }^{19 \sim 22)}$ Also, OKAnishi and KINAMI reported the restoration of kasugamycin production by citrulline in a Streptomyces kasugaensis mutant with impaired arginine synthesis. ${ }^{23)}$

Bicozamycin (synonym: Bicyclomycin) is a cyclic peptide antibiotic which consists of oxidized leucine and isoleucine ${ }^{24)}$ and it is commercially important as a feed-additive due to its bactericidal effect on enteric bacteria. Previously, OCHI et al. showed that bicozamycin production and aerial mycelium formation by Streptomyces griseoflavus, wild type strain 1805, were blocked simultaneously by a mutation to arginine auxotrophy. Both losses could be reversed completely by addition of citrulline. ${ }^{10)}$ The present report deals with the influence of the stringent response (ppGpp) and ornithine cycle for the initiation of secondary metabolism of $S$. griseoflavus strain 1805 and a bicozamycin high-producing strain derived from it. 


\section{Materials and Methods}

\section{Strains}

S. griseoflavus FERM 1805 is a prototrophic, bicozamycin-producing wild type strain. S. griseoflavus 006 is a bicozamycin high-producing strain derived from strain 1805 by sequential mutagenic treatments ( $>15$ times). The latter strain produces bicozamycin more than 300 times as much amount as the former.

\section{Growth Conditions}

MG medium, a complex medium for bicozamycin production, contained glucose $10 \mathrm{~g}$, Polypepton $8 \mathrm{~g}$, yeast extract $5 \mathrm{~g}, \mathrm{KH}_{2} \mathrm{PO}_{4} 0.5 \mathrm{~g}, \mathrm{MgSO}_{4}$ (anhydrous) $0.3 \mathrm{~g}, \mathrm{NaCl} 0.5 \mathrm{~g}$ and $1 \mathrm{M} \mathrm{MOPS}$ $100 \mathrm{ml}$ (adjusted to $\mathrm{pH} 6.0$ with $\mathrm{KOH}$ ) in 1 liter deionized water. The composition of a 1) synthetic medium, 2) glucose - Polypepton - yeast extract medium (GPY medium), and 3) glucose - yeast extract - malt extract agar (GYM agar) was described previously. ${ }^{10)}$

A monoconidial suspension of each strain, prepared by scraping the spores from a GYM agar slant and blending in a vortex mixer with glass beads, was inoculated into GPY medium $(50 \mathrm{ml} / 250-\mathrm{ml}$ Erlenmeyer flask), which was incubated at $30^{\circ} \mathrm{C}$ on a rotary shaker $(250 \mathrm{rpm})$. After 30 hours incubation, cells $(1 \mathrm{ml})$ were inoculated into $\mathrm{MG}$ medium $(50 \mathrm{ml} / 250-\mathrm{ml}$ Erlenmeyer fiask), and cultivated at $30^{\circ} \mathrm{C}$ on a rotary shaker $(250 \mathrm{rpm})$. Cells, grown in GPY medium for 30 hours and previously washed in saline, were inoculated into synthetic medium supplemented with vitamin-free Casamino acids, as described earlier. ${ }^{10)}$

\section{Bicozamycin Resistance}

Cells of each strain, grown in GPY medium for 30 hours, were inoculated at a $1: 200$ dilution into MG medium containing various amounts of bicozamycin. Growth was observed after 24 hours of incubation with shaking.

\section{Assay of Nucleotide Pool}

Nucleotide pools were assayed by means of HPLC after extraction of mycelium with $1 \mathrm{M}$ formic acid, as described previously. ${ }^{10,25)}$ The intracellular pool size of nucleotides is expressed as pmol or nmol per mg (dry weight) of cells.

\section{Assay of Amino Acid Pool}

Cells from 10 to $100 \mathrm{ml}$ culture were quickly harvested by filtration (filter-paper diameter $9 \mathrm{~cm}$, No. 2; Toyo Roshi Co.), extracted with $10 \mathrm{ml}$ of $0.3 \mathrm{M}$ perchloric acid plus $1 \mathrm{~mm}$ EDTA as described, ${ }^{20}$ ) and then $50 \mu \mathrm{l}$ aliquots were applied to an amino acid analyzer (Hitachi Amino Acid Analyzer; Type 835). Amino acids in the pool are expressed as nmol per mg (dry weight) of cells.

\section{Assay of Ornithine Transcarbamylase (EC 2.1.3.3)}

A crude enzyme preparation was obtained by sonic disruption ( $c a .2$ minutes) of cells ( $2 \mathrm{~g}$ wet weight) suspended in $5 \mathrm{ml}$ of $10 \mathrm{mM}$ Tris $-\mathrm{HCl}$ buffer ( $\mathrm{pH} 7.5$ ). The cellular debris was removed by centrifugation at $15,000 \times g$ for 20 minutes at $3^{\circ} \mathrm{C}$. The supernatant, containing 5 to $15 \mathrm{mg}$ of protein per $\mathrm{ml}$, was used as the enzyme preparation. Ornithine transcarbamylase activity was determined as described by NAKamura and Jones. ${ }^{27}$ ) The reaction mixture contained $50 \mu \mathrm{l}$ of $1 \mathrm{M}$ Tris $-\mathrm{HCl}$ buffer (pH 8.5), $25 \mu \mathrm{l}$ of $0.1 \mathrm{M}$ dilithium carbamyl phosphate, $25 \mu 1$ of $0.1 \mathrm{M} \mathrm{L}$-ornithine $\cdot \mathrm{HCl}, 5 \sim 20 \mu 1$ of the enzyme preparation, and deionized water to a final volume of $0.5 \mathrm{ml}$. The reaction was initiated by addition of ornithine and incubation was run at $37^{\circ} \mathrm{C}$ for 15 minutes. The reaction was stopped by addition of $1.5 \mathrm{ml}$ of $0.25 \mathrm{~N}$ trichloroacetic acid. Citrulline produced in the reaction mixture was measured colorimetrically as described by OGINSKY. ${ }^{28)}$ Specific activity is expressed as $\mu \mathrm{mol}$ of citrulline formed per hour per mg protein. Protein was assayed by the method of Lowry et al. ${ }^{29)}$

\section{Assay of Bicozamycin}

The bicozamycin titer was determined by a disc-plate method with Escherichia coli strain BS-10 (a bicozamycin-sensitive strain) as a test organism. The assay plate (diameter $8.7 \mathrm{~cm}$ ) contained $10 \mathrm{ml}$ of Mueller-Hinton medium plus $0.8 \%$ agar. The bicozamycin titer was also determined by means of HPLC as follows: A 10- $\mu$ l sample (supernatant of the cultured broth) was applied directly to a column 
(Cosmosil packed column, $4.6 \times 150 \mathrm{~mm}$, Code No. 39047, Nakarai Chemicals Co.) and bicozamycin was eluted at retention time of 8.4 minutes with a solution containing $0.2 \%$ tetrahydrofuran and $15 \mathrm{~mm}$ phosphoric acid at a flow rate of $1.5 \mathrm{ml} /$ minute and absorbance was measured at $210 \mathrm{~nm}$.

\section{Results}

Bicozamycin Production by the Wild Type and High-producing Strains

Bicozamycin synthesis $(60 \mu \mathrm{g} / \mathrm{ml})$ by the wild type strain in a chemically defined medium was

Fig. 1. The time course of bicozamycin production and changes in ppGpp, GTP, and ATP pools of Streptomyces griseoflavus strains cultivated in MG medium.

Wild type strain 1805, bicozamycin high-producing strain 006, - growth, --- bicozamycin.

(A)

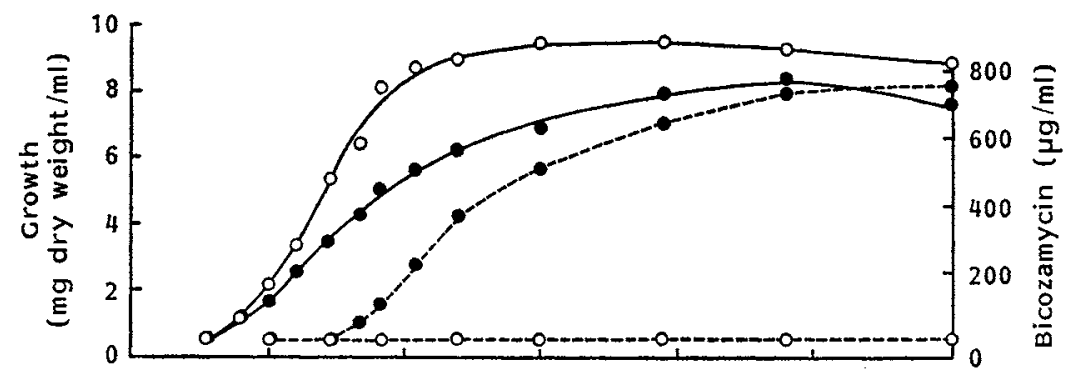

(B)

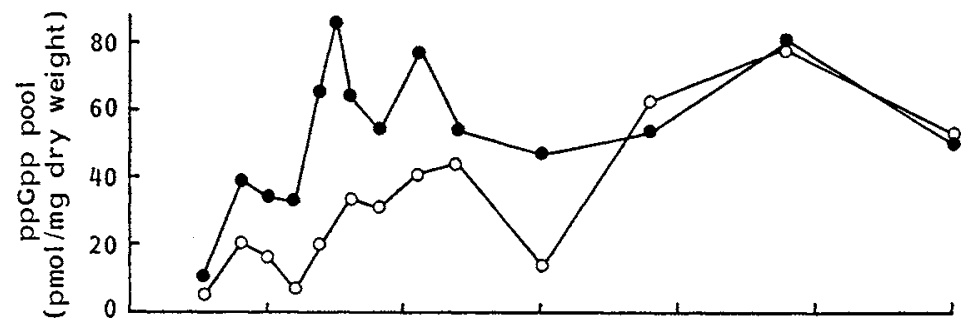

(c)

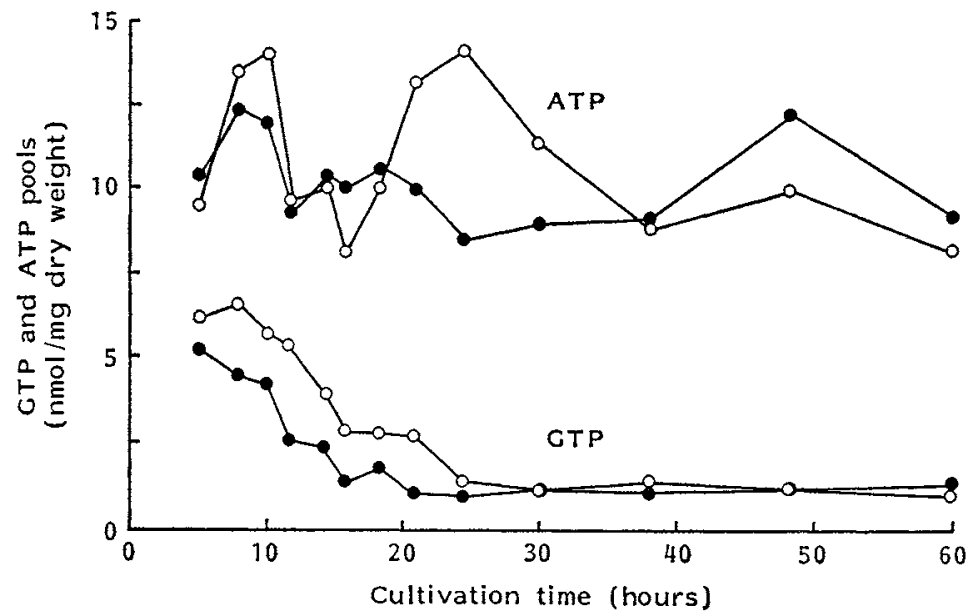

All the experimental procedures were described in Materials and Methods. The data were all from the same experiment. 
inhibited completely $(<0.5 \mu \mathrm{g} / \mathrm{ml})$ by addition of $2 \%$ (weight/volume) Casamino acids, in similar to the other antibiotic fermentations by Streptomyces $\mathrm{sp}^{14 \sim 18)}$ In addition, the wild type strain produced only $1 \sim 2 \mu \mathrm{g} / \mathrm{ml}$ of bicozamycin in $\mathrm{MG}$ medium, a nutritionally rich medium containing $0.8 \%$ Polypepton. In contrast, the high-producing strain produced a large amount of bicozamycin $(800 \mu \mathrm{g} / \mathrm{ml})$ in $\mathrm{MG}$ medium. Its formation was initiated at 14 hours after inoculation and rapid synthesis of bicozamycin was observed within a short period of time (10 hours) (Fig. 1A). Thereafter the rate of antibiotic synthesis declined significantly. This may be due to a limitation of precursors per se required for bicozamycin synthesis; addition of both leucine and isoleucine, known precursors for bicozamycin synthesis, ${ }^{24)}$ at 28 hours, markedly stimulated antibiotic production, without affecting the growth rate or yield of biomass (Fig. 2). Thus, the availability of a larger supply of precursors (including indirect precursors) appeared to be an essential factor for high productivity of the antibiotic. We, therefore, assumed that the
Fig. 2. Bicozamycin production by strain 006 .

- Plus leucine and isoleucine, $O$ control.

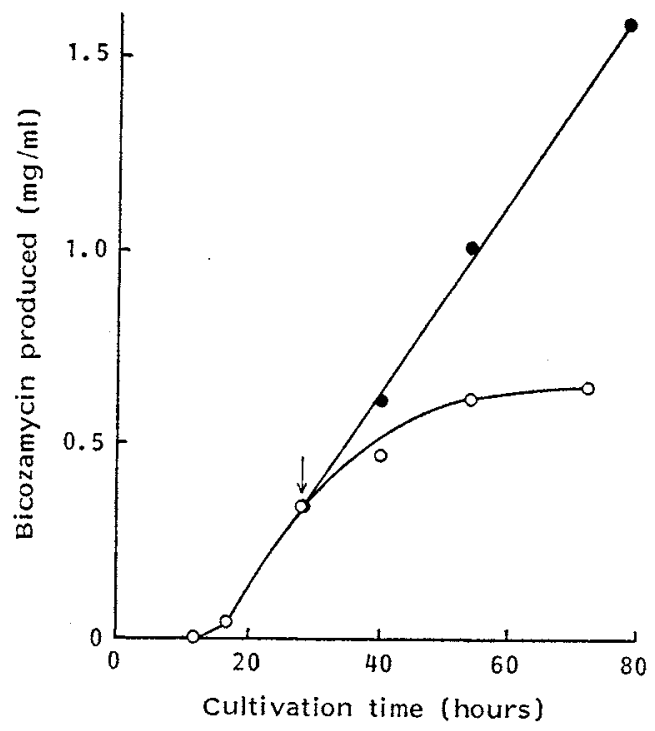

Cells of strain 006 were grown in the same medium as in Fig. 1. After 28 hours of incubation the culture was divided into two flasks and leucine and isoleucine were added to one of them each at final concentration of $0.2 \%$, and then incubation was further continued for 72 hours. The arrow indicates the time of addition of leucine and isoleucine.

high-producing strain may harbor genetic factor(s) responsible for a greater pool size of amino acids, especially leucine and isoleucine. This was confirmed by using cells harvested at various growth phase. As shown in Table 1, the high-producing strain maintained the total amino acid pool size $20 \sim 70 \%$ higher than those of the wild type strain throughout the cultivation. The larger pool for amino acids not just leucine and isoleucine in the former than the latter may contribute to the greater synthesis of bicozamycin as a source of indirect precursors. The pool sizes of leucine and isoleucine in the former were 3- to 6-fold higher than the later after 8 hours cultivation. Later (e.g. 24 hours), the pool sizes of leucine and isoleucine (and some other amino acids) of the former were even lower than the latter. This may be explained by a rapid utilization of those amino acids for bicozamycin synthesis. Since the wild type strain still failed to produce bicozamycin even with leucine and isoleucine supplementation it is suggested that the high-producing strain may also harbor mechanism(s) by which 'initiation processes' of the antibiotic synthesis are accelerated.

\section{Nucleotide Pool Changes during Cultivation}

It has been postulated that ppGpp may be a signal molecule which triggers (or is needed for) antibiotic synthesis. ${ }^{14 \sim 16)}$ We, therefore, measured the changes in ppGpp pool size during cultivation. As shown in Fig. 1B, the pool size of ppGpp (strain 1805) increased gradually and was maximal at 24 hours when cells had just entered the stationary phase. Interestingly, the ppGpp pool size of the highproducing strain 006 was about 2- to 3-fold greater than that of the wild type strain 1805 . It should 
Table 1. Amino acid pools of strains 1805 and 006 grown in MG medium.

Cells cultivated for indicated times were harvested and extracted for assay of amino acid pools. The results are from the same experiment in Fig. 1.

\begin{tabular}{|c|c|c|c|c|c|c|}
\hline \multirow{3}{*}{$\begin{array}{l}\text { Amino } \\
\text { acid }\end{array}$} & \multicolumn{6}{|c|}{ Amino acid pools (nmol/mg dry weight) } \\
\hline & \multicolumn{2}{|c|}{8 hours } & \multicolumn{2}{|c|}{14 hours } & \multicolumn{2}{|c|}{24 hours } \\
\hline & 1805 & 006 & 1805 & 006 & 1805 & 006 \\
\hline $\mathrm{Aba}^{\mathrm{a}}$ & 7.05 & 14.3 & 3.79 & 6.97 & 0.64 & 0.38 \\
\hline Ala & 9.48 & 5.35 & 3.31 & 2.38 & 1.89 & 3.70 \\
\hline Arg & 0.93 & 2.39 & 0.37 & 0.57 & 0.20 & 0.29 \\
\hline Asp & 1.73 & ND & 4.18 & 3.13 & 2.71 & 3.16 \\
\hline Cys & 0.42 & 0.48 & 0.33 & 0.28 & 0.52 & 1.11 \\
\hline $\mathrm{Dap}^{\mathrm{b}}$ & 0.85 & 1.13 & 0.37 & 0.60 & 1.47 & 13.2 \\
\hline Gln & 31.5 & 76.1 & 51.5 & 39.1 & 23.7 & 35.4 \\
\hline Glu & 117 & 142 & 136 & 177 & 83.0 & 151 \\
\hline Gly & 0.25 & 5.50 & 0.73 & 3.10 & $\mathrm{ND}$ & 0.13 \\
\hline His & 0.41 & 1.17 & 0.10 & 0.19 & 0.09 & $\mathrm{ND}$ \\
\hline Ile & 0.67 & 4.01 & 0.47 & 0.63 & 0.51 & 0.14 \\
\hline Leu & 4.92 & 15.3 & 1.58 & 3.94 & 0.70 & 0.52 \\
\hline Lys & 1.21 & 4.06 & 4.26 & 9.66 & 1.95 & 0.54 \\
\hline Met & 3.10 & 4.78 & 2.75 & 3.46 & 0.51 & 0.28 \\
\hline Orn & 0.21 & 0.79 & 0.41 & 0.80 & 0.66 & 0.40 \\
\hline Phe & 1.52 & 3.38 & 2.46 & 3.22 & 0.64 & 0.21 \\
\hline Ser & 0.94 & 2.82 & 1.41 & 1.43 & 3.18 & 1.09 \\
\hline Val & 2.25 & 17.3 & 7.10 & 5.69 & 1.03 & 0.81 \\
\hline Total & 184 & 301 & 221 & 262 & 123 & 212 \\
\hline
\end{tabular}

a $\gamma$-Amino butyric acid. b $\alpha, \varepsilon$-Diaminopimelic acid.

ND: Not detected.

be pointed out that the onset of bicozamycin production (Fig. 1A) appears to coincide to the maximal accumulation of ppGpp. A decrease in the GTP pool size (Fig. 1C) occurred concomitantly with increase in ppGpp. No marked difference between the wild type and high-producing strains was observed in the pool size of ATP (Fig. 1C), UTP or CTP (not shown). The accumulation of ppGpp at higher levels in the mutant was also observed by the experiment using MG medium containing as much as $2 \%$ Polypepton. Thus, it is suggested that the high-producing strain, as a consequence of one or more mutational events, has the ability to accumulate higher levels of ppGpp.

We also investigated how $S$. griseoflavus accumulated ppGpp. When the wild type strain, grown to the mid-exponential growth phase in the chemically defined medium supplemented with $2 \%$ Casamino acids, was transferred to the fresh chemically defined medium without Casamino acids, there was an immediate accumulation of ppGpp after shift-down (Fig. 3). Apparently, S. griseoflavus, like other Streptomyces sp., ${ }^{1416)}$ accumulates ppGpp in response to amino acid starvation. Later, i.e., $2 \sim 4$ hours after transfer, ppGpp pool size decreased (though still higher than the initial level), possibly due to adaptation of cells to de novo synthesis of amino acids. Similar experiments was not applicable to the high-producing strain because it grew too slowly in the Casamino acid medium mentioned above.

\section{Ornithine Transcarbamylase Activity during Cultivation}

It has been postulated that citrulline positively controls the initiation of bicozamycin production. ${ }^{10)}$ We investigated further the putative role of this amino acid in the high-producing strain. 
Fig. 3. Changes in the intracellular pools of nucleoside triphosphates and ppGpp after nutritional shiftdown.

$\triangle$ ppGpp, $\bullet$ GTP, $\bigcirc$ ATP, $\square$ UTP, $\triangle$ CTP.

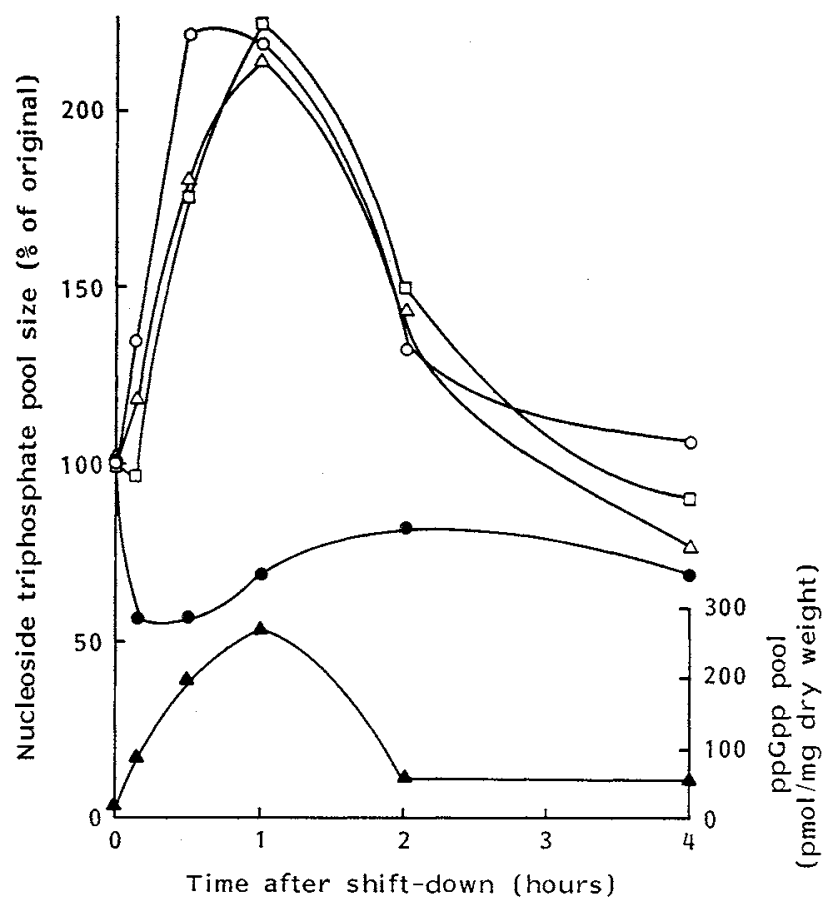

Cells of strain 1805 were grown to mid-exponential phase, 10 hours after inoculation, in the chemically defined medium supplemented with $2 \%$ (weight/volume) vitamin-free Casamino acids and transferred to the fresh chemically defined medium without Casamino acids. Nucleotide pools were determined as described in Materials and Methods. Just before transfer, nucleotide pools of GTP, ATP, UTP and CTP were $3,430,9,250,3,950$ and $2,770 \mathrm{pmol} / \mathrm{mg}$ dry weight, respectively.

In similar to previous report for the wild type strain, ${ }^{10)}$ the citrulline pool size was also undetectable for strain 006. We, therefore, analyzed the dynamic aspect of the synthesis of ornithine transcarbamylase (OTCase) which produces citrulline from ornithine and carbamyl phosphate. In both strains, specific activity of OTCase was quite low in young cultures, but its synthesis was abruptly derepressed at 10 hours (Fig. 4) as similarly reported for Bacillus subtilis. ${ }^{30)}$ Derepression of OTCase formation, particularly striking in the high-producing strain, was about 4-fold greater than that observed in the wild type strain (Fig. 4). Interestingly, the derepression of enzyme synthesis took place just prior to initiation to bicozamycin production. Since pool size of the substrate (ornithine) of the high-producing strain at 14 hours was greater than that of the wild type strain (Table 1), the former strain could produce more than 4-fold amount of citrulline at this time.

\section{Characterization of Ornithine Transcarbamylase}

In the wild type strain, OTCase was repressed completely to the basal level when cells were grown in MG medium supplemented with $1 \mathrm{~mm}$ arginine (Fig. 5). In contrast, the high-producing strain produced OTCase at high levels in the medium containing up to $2 \mathrm{mM}$ arginine, but its production was fully repressed to basal levels by addition of $5 \mathrm{~mm}$ arginine. Thus, OTCase synthesis in the highproducing strain is partially, but not completely, desensitized to end-product repression. Although 
Fig. 4. Changes in specific activity of ornithine transcarbamylase (OTCase) during growth in MG medium.

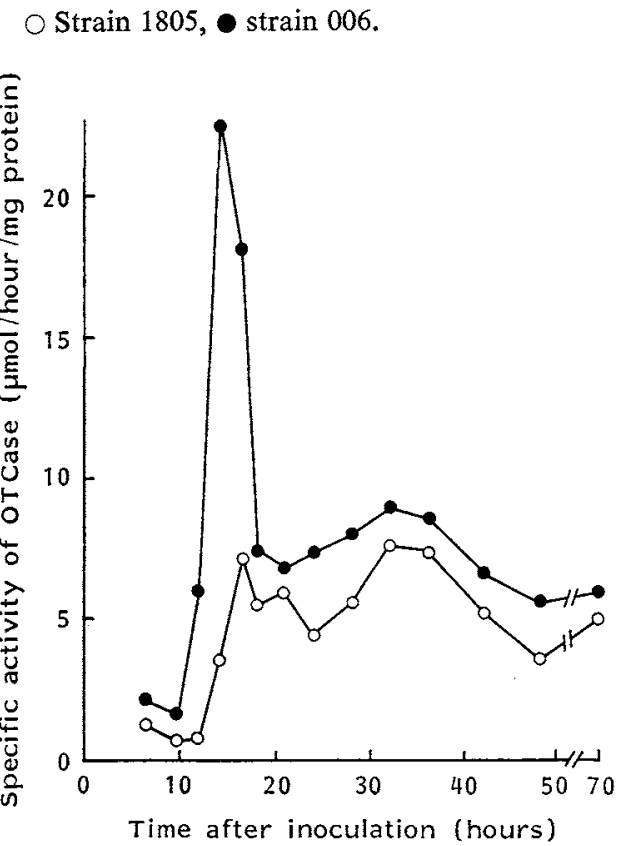

OTCase of the wild type strain was also subject to inhibition by arginine, its inhibition was weak, i.e. $38 \%$ inhibition by $10 \mathrm{~mm}$ arginine in the presence of $1 \mathrm{~mm}$ ornithine.

\section{Ability to form Aerial Mycelium}

Both the wild type and high-producing strains developed aerial mycelium after 3 days of inoculation on GYM agar. Since the GTP pool size decreased more rapidly in the high-producing than in the wild type strain as examined in the liquid culture (Fig. 1C), it was reasoned that the high-producing strain might exhibit a greater ability to form aerial mycelium. ${ }^{31}$ As expected, development of aerial mycelium by the wild type strain was completely inhibited during 10 days' incubation when cells were grown on GYM agar supplemented with $4 \%$ Casamino acids; in contrast, the high-producing strain still produced aerial mycelium even with $5 \%$ Casamino acids in
Fig. 5. Repression of ornithine transcarbamylase (OTCase) by arginine.

Strain 1805 , strain 006.

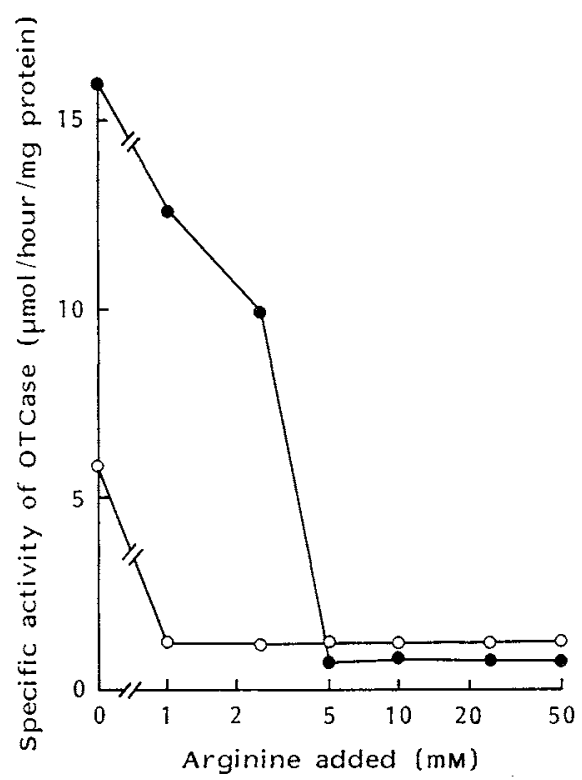

Cells of strains 1805 and 006 were grown in MG medium supplemented with various amounts of arginine, and OTCase activity was determined after 16 hours of cultivation.

Fig. 6. Reversal of aerial mycelium formation by addition of decoyinine in Streptomyces griseoflavus strain 1805 on a plate containing excess amount of Casamino acids.

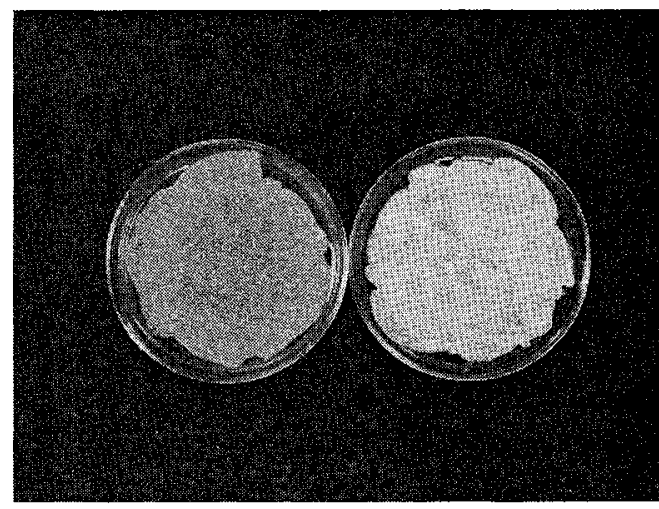

Spores were spread on GYM agar (plus $100 \mathrm{~mm}$ MOPS, pH 7.0 with $\mathrm{KOH}$ ) supplemented with $4 \%$ vitamin-free Casamino acids without (left) or with (right) $0.3 \mathrm{~mm}$ decoyinine. The plates were incubated at $30^{\circ} \mathrm{C}$ for 7 days.

the medium. Complete inhibition was only observed with $7 \%$ Casamino acids. However, Casamino acid-suppressed culture of the wild type strain was able to induce formation of aerial mycelium and also 
eventually to sporulate by addition of $0.1 \sim 0.3 \mathrm{~mm}$ decoyinine, a specific inhibitor of GMP synthetase (Fig. 6), in similar to that seen with other Streptomyces sp. ${ }^{16,312}$

\section{Resistance to Bicozamycin}

Resistance of $S$. griseoflavus to bicozamycin (self-resistance) was also examined. The highproducing strain showed 5 -fold greater resistance than the wild type strain $(10 \mathrm{mg} v \mathrm{vs} .2 \mathrm{mg} / \mathrm{ml}$ of bicozamycin to attain a $50 \%$-growth inhibition, respectively).

\section{Discussion}

On the contrary to the impaired ability of micro-organisms to produce antibiotics caused by rel mutation or mutation to arginine auxotrophy, it was shown in this study that a dramatically increased ability to produce bicozamycin was associated with increase in specific activity of ornithine transcarbamylase and accumulation of ppGpp. The high-producing strain is the mutant strain carefully selected to the level of industrial use. Therefore, we suggest that the observed physiological properties of the high-producing strain are a consequence of the mutations and strain selections and might be responsible for the enhanced production of bicozamycin. Although this hypothesis is an attractive one, the results described herein do not provide an absolute correlation between them. The mechanism of initiation of antibiotic production may involve certain regularoty systems or processes. Thus, regulatory mutations could result in an acceleration of transcriptional events leading to initiation and expression of biosynthetic genes of the antibiotic. From these points of view, we have previously shown that the introduction of relaxed (rel) mutations into Bacillus subtilis (2 strains) and Streptomyces ( 3 species) results in loss of the ability to produce antibiotics. ${ }^{14 \sim 18,32)}$ As will be reported elsewhere, a rel mutant derived from $S$. griseoflavus wild type strain, obtained as a spontaneous thiopeptin-resistant isolates, also lost the ability to produce bicozamycin. From the study of stepwise enhancement of $\alpha$-amylase production, MARUO et al. emphasized the importance of synergistic rather than additive effects of the pre-existing and added genetic factors. ${ }^{33,34)}$ In the light of these findings one should recognize with respect to strain improvement that each genetic factor can exert its maximal effect only through interactions with other genetic factors.

An increase in ability to accumulate intracellular ppGpp could arise from either its increased synthesis or decreased degradation, or a combination of these two processes. A ppGpp is synthesized in response to amino acid starvation by the action of $\mathrm{relA}$ gene product (stringent factor) and uncharged tRNA, using ATP and GTP as substrates. ${ }^{35)}$ Increased synthesis of ppGpp, therefore, could arise from mutations which depress transport of amino acids or its de novo synthesis, or from those which interfere with amino acid-charging of tRNA, or from those which alter ribosomal machinery per se. On the other hand, decreased degradation could result from mutations which depress activity of protein(s) involved in degradation. Such a mutation, designated $s p o T$, is known in $E$. coli. ${ }^{35\rangle}$ Since turnover of $\mathrm{ppGpp}$ is very rapid, 20 seconds in $E$. coli ${ }^{36)}$ the impeded degradation may be very effective for increase in ppGpp accumulation. Indeed, a spoT mutant was shown to maintain 2- to 3-fold higher concentration of ppGpp than a spoT $T^{+}$strain..$^{36)}$ In addition to the well-known mechanism of ppGpp synthesis described above, ribosome-independent or stringent factor-independent ppGpp synthesis is also known. ${ }^{37 \sim 39}$ It remains unknown whether such a novel type of ppGpp synthesis contributed or not to the observed increase in the ppGpp pool size during cultivation of the highproducing strain. The mechanism by which the ppGpp pool size of the wild type strain increased during late stationary phase (Fig. 1B) also remains obscure. However, contribution of the increased ppGpp pool for initiation of the antibiotic production may be negated in this strain by the limited nutrient and precursor pools.

The most important thing for obtaining readily a desired mutant is the availability of a selection procedure(s) or facile detection method. In this regard, the increased ability of the high-producing strain to form aerial mycelium may offer an intriguing possibility as a screening procedure. Conceivably, aerial mycelium formation may partially be due to a severe inhibition of GMP (and GTP) 
synthesis by ppGpp, which leads to the onset of differentiation. This hypothesis is consistent with the facts that Casamino acid-repression of aerial mycelium formation was completely reversed by addition of decoyinine (Fig. 6) and that a S. griseoflavus rel mutant showed a reduced and dramatically delayed appearance of aerial mycelium (unpublished). Therefore, mutants with increased ability to accumulate ppGpp may be detected at high frequency by selection of colonies which develop aerial mycelium on nutritionally rich media.

\section{References}

1) ENSIGN, J. C.: Developmental biology of actinomycetes. In Overproduction of Microbial Metabolites. FEMS Symposium 13. Ed., V. KRumphanzL et al., pp. 127 140, Academic Press, London, 1982

2) Chater, K. F.: Morphological and physiological differentiation in Streptomyces. In Microbial Development. Eds., R. Losick \& L. ShAPIRo, pp. 89 116, Cold Spring Harbor Laboratory, New York, 1984

3) Kalakoutskin, L. V. \& N. S. AGRE: Comparative aspects of development and differentiation in actinomycetes. Bacteriol. Rev. 40: 469 524, 1976

4) MATEJU, J.; E. CuRdova, V. JECHOVA \& Z. VANeK: Importance of nutrient limitation in the biosynthesis of secondary metabolites. In Biological, Biochemical and Biomedical Aspects of Actinomycetes. Ed., G. SzaBo et al., pp. $171 \sim 176$, Akademiai Kiado, Budapest, 1986

5) KнокнLov, A.S.: Actinomycete autoregulators. In Biological, Biochemical and Biomedical Aspects of Actinomycetes. Ed., G. SzABo et al., pp. 791 798, Akademiai Kiado, Budapest, 1986

6) HARA, O. \& T. BEPPU: Mutants blocked in streptomycin production in Streptomyces griseus - the role of A-factor. J. Antibiotics 35: 349 358, 1982

7) GräFe, U.; G. Reinhardt, D. Krebs, I. ERItT \& W. F. Fleck: Pleiotropic effects of a butyrolactonetype autoregulator on mutants of Streptomyces griseus blocked in cytodifferentiation. J. Gen. Microbiol. 130: $1237 \sim 1245,1984$

8) Vitalis, S.; G. Valu, I. BeKesi, F. Szeszak \& G. Szabo: Effect of factor C on the differentiation of Streptomyces griseus. In Biological, Biochemical and Biomedical Aspects of Actinomycetes. Ed., G. SzaBo et al., pp. 799 806, Akademiai Kiado, Budapest, 1986

9) Redshaw, P. A.; P. A. McCann, M. A. Pentella \& B. M. Pogell: Simultaneous loss of multiple differentiated functions in aerial mycelium-negative isolates of streptomycetes. J. Bacteriol. 137: 891 899,1979

10) Ochi, K.; Y. SAIto, K. Umehara, I. Ueda \& M. KohsaKa: Restoration of aerial mycelium and antibiotic production in a Streptomyces griseoflavus arginine auxotroph. J. Gen. Microbiol, 130: 2007 2013, 1984

11) Ogawara, H.; H. Ishihara \& M. M. NaKano: Cloning of argG complementary gene from Streptomyces species. In Biological, Biochemical and Biomedical Aspects of Actinomycetes. Ed., G. SzABo et al., pp. 329 334, Akademiai Kiado, Budapest, 1986

12) Merrick, M. J.: A morphological and genetic mapping study of bald colony mutants of Streptomyces coelicolor. J. Gen. Microbiol. 96: 299 315, 1976

13) NoACK, D. \& M. Rотн: Directed selection of differentiation mutants by aid of chemostat cultivation. In Biological, Biochemical and Biomedical Aspects of Actinomycetes. Ed., G. SzABo et al., pp. 807 816, Akademiai Kiado, Budapest, 1986

14) OcH, K.: Occurrence of the stringent response in Streptomyces sp. and its significance for the initiation of morphological and physiological differentiation. J. Gen. Microbiol. 132: 2621 2631, 1986

15) OCHI, K.: A rel mutation abolishes the enzyme induction needed for actinomycin synthesis in Streptomyces antibioticus. Agric. Biol. Chem. 51: 829 835, 1987

16) OcHI, K.: Metabolic initiation of differentiation and secondary metabolism by Streptomyces griseus: Significance of the stringent response (ppGpp) and GTP content in relation to A factor. J. Bacteriol. 169: 3608 3616, 1987

17) OcHI, K.: Changes in nucleotide pools during sporulation of Streptomyces griseus in submerged culture. J. Gen. Microbiol. 133: 2787 2795, 1987

18) Coleman, R. H. \& J. C. Ensign: Regulation of formation of aerial mycelia and spores of Streptomyces viridochromogenes. J. Bacteriol. 149: 1102 1111, 1982

19) VARGHA, G.; T. Karsal \& G. Szabó: A conditional aerial mycelium-negative mutant of Streptomyces fradiae with deficient ornithine carbamoyltransferase activity. J. Gen. Microbiol. 129: 539 542, 1983

20) VARGHA, G. \& G. SZABO: Nutritional requirements for differentiation in a Streptomyces fradiae mutant. Z. Allg. Mikrobiol. 24: 189 199, 1984 
21) VARgha, G.; V. Zs.-NAGY, G. LustYIK \& G. SzaBó: Methionine requirement of sporulation in a Streptomyces fradiae mutant. J. Gen. Microbiol. 132: 2931 2936, 1986

22) VARGHA, G. \& G. SzABo: Metabolic control of differentiation in Streptomyces fradiae. In Biological, Biochemical and Biomedical Aspects of Actinomycetes. Ed., G. SzABo et al., pp. 835 837, Akademiai Kiado, Budapest, 1986

23) Okanishi, M. \& T. Kinami: Positive regulation of antibiotic production with citrulline. In Biological, Biochemical and Biomedical Aspects of Actinomycetes. Ed., G. SzABo et al., pp. 851 853, Akademiai Kiado, Budapest, 1986

24) Iseki, M.; T. MiYoshi, T. Konomi \& H. ImANAKA: Biosynthesis of bicyclomycin. II. Biosynthetic conditions and incorporation of radioactive precursors into bicyclomycin by washed mycelium. J. Antibiotics 33: $488 \sim 493,1980$

25) OCHI, K.; J. C. KANDala \& E. FreESE: Initiation of Bacillus subtilis sporulation by the stringent response to partial amino acid deprivation. J. Biol. Chem. 256: 6866 6875, 1981

26) OCHI, K. \& E. FREESE: A decrease in $S$-adenosylmethionine synthetase activity increases the probability of spontaneous sporulation. J. Bacteriol. 152: $400 \sim 410,1982$

27) Nakamura, M. \& M. E. Jones: Ornithine carbamyltransferase (Streptococcus faecalis). Methods Enzymol. 17A: 287 294, 1971

28) OGINSKy, E. L.: Isolation and determination of arginine and citrulline. Methods Enzymol. 3: 639 643,1957

29) Lowry, O. H.; N. J. Rosebrough, A. L. Farr \& R. J. Randall: Protein measurement with the Folin phenol reagent. J. Biol. Chem. 193: 265 275, 1951

30) Deutscher, M.P. \& A. Kornberg: Biochemical studies of bacterial sporulation and germination. VIII. Patterns of enzyme development during growth and sporulation of Bacillus subtilis. J. Biol. Chem. 243: $4653 \sim 4660,1968$

31) OCHI, K.: A decrease in GTP content is associated with aerial mycelium formation in Streptomyces MA406-A-1. J. Gen. Microbiol. 132: 299 305, 1986

32) OCHI, K. \& S. OHSAwA: Initiation of antibiotic production by the stringent response of Bacillus subtilis Marburg. J. Gen. Microbiol. 130: 2473 2482, 1984

33) Maruo, B.; K. Yamane, Y. Yoneda \& K. Hrtotsuryanagi: Stepwise genetic transformation of Bacillus subtilis with enhancement of productivity of $\alpha$-amylase. Proc. Jpn. Acad. 54: 435 439, 1978

34) Maruo, B. \& T. Tojo: Stepwise enhancement of productivity of thermostable amylase in Bacillus licheniformis by a series of mutations. J. Gen. Appl. Microbiol. 31: 323 328, 1985

35) Gallant, J. A.: Stringent control in E. coli. Annu. Rev. Genet. 13: 393 415, 1979

36) Stamminger, G. \& R. A. Lazzarini: Altered metabolism of the guanosine tetraphosphate, ppGpp, in mutants of E. coli. Cell 1: 85 90, 1974

37) Nishino, T. \& S. Murao: Possible involvement of plasmid in nucleotide pyrophosphokinase production and the relationship between this productivity and cellular accumulation of guanosine tetraphosphate (ppGpp) in Streptomyces. Agric. Biol. Chem. 45: 199 208, 1981

38) Biro, S.; S. ViTalis \& G. SzaBo: Detection and changes of nucleotide pyrophosphotransferase activity in cultures of Streptomyces griseus strains on solid media. Acta Biol. Acad. Sci. Hung. 30: 381 386, 1979

39) Ikehara, K.; H. OKada, K. Maeda, A. Ogura \& K. Sugae: Accumulation of rela gene-independent ppGpp in Bacillus subtilis vegetative cells upon temperature shift-down. J. Biochem. 95: 895 897, 1984 